\title{
BIFURCATIONS AND LIMIT CYCLES IN A MODEL FOR A VOCAL FOLD OSCILLATOR*
}

\author{
JORGE C. LUCERO ${ }^{\dagger}$
}

\begin{abstract}
This article presents an analysis of the dynamics of a bidimensional oscillator, which has been proposed as a simple model for the vocal fold motion at phonation. The model is capable of producing an oscillation with physiologically realistic values for the parameters. A simple extension of the model using even-powered polynomials in the damping factor is proposed, to permit the occurrence of an oscillation hysteresis phenomenon commonly observed in voice onset-offset patterns. This phenomenon appears from the combination of a subcritical Hopf bifurcation where an unstable limit cycle is produced, with a fold bifurcation between limit cycles, where the unstable limit cycle coalesces and cancels with a stable limit cycle. The results are illustrated with phase plane plots and bifurcation diagrams obtained using numerical continuation techniques.
\end{abstract}

Key words. Bifurcation, limit cycle, oscillation, vocal folds.

AMS subject classifications. 34C15, 34C23, 34C25, 34C60, 37G15, 70K42, 92C10

\section{Introduction}

The vocal folds at the larynx constitute a biomechanical oscillator that acts as the sound source in phonation. Under certain conditions of instability relating parameters of their physiology and associated airway aerodynamics, the airflow that passes through the channel between the folds (glottis) induces their oscillation. The vocal fold oscillation in turn modulates the glottal airflow, which after going through the oral and nasal cavities, results in the air pressure wave that we perceive as voice [1]. Thus, it is a self-excited flow-induced oscillation, a similar phenomenon to the production of sound at the syrinx of songbirds [2], at blood vessels during sphygmomanometry [3], and at the lips in brass musical instruments [4].

Since the pioneering work by Van den Berg et al. [5] which set the basis of glottal aerodynamics, several mathematical models have been proposed to characterize the dynamics of this oscillator. Such models have ranged in complexity from the simple and popular one-mass [6] and two-mass [7] models, in which each vocal fold is represented by one or two coupled mass-damper-spring systems, respectively, up to continuum models and finite element approaches [8]. Although the more sophisticated models are needed to capture subtle details of vocal fold motion and laryngeal aerodynamics $[8,9]$, simple low-dimensional models are still useful to analyze the underlying dynamics of the oscillation. In fact, simple models have shown a rich variety of interesting nonlinear phenomena, including the existence of multiple equilibrium positions and limit cycles [10, 11], several types of bifurcations [10, 11, 12], and chaotic behavior $[11,13]$.

A particularly useful model has been Titze's mucosal wave model [14]. There, the movement of the vocal fold tissues is represented as a surface wave propagating in the direction of the airflow. The model was originally conceived for small amplitude oscillations, to study conditions for the onset of the oscillation. An extension to the general case of large amplitude oscillations was proposed later [15, 10], by using

\footnotetext{
*Received: June 22, 2005; accepted (in revised version): September 23, 2005. Communicated by Jack Xin.

This work was supported by CT-Info/MCT/CNPq (Brazil).

${ }^{\dagger}$ Department of Mathematics, University of Brasilia, Brasilia DF 70910-900, Brazil (lucero@ mat.unb.br).
} 
a more detailed description of the glottal aerodynamics which included the effect of formation of an air jet downstream from a divergent glottal channel. Using this extension, the existence of Appleton and van der Pol's oscillation hysteresis phenomenon [16] was shown. This phenomenon appears from the combination of a subcritical Hopf bifurcation where an unstable limit cycle is produced, with a fold bifurcation between limit cycles, where the unstable limit cycle coalesces and cancels with a stable limit cycle. It is also known as hard-excited oscillation [17], in contrast to the soft-excited case, which corresponds to the supercritical type of the Hopf bifurcation. Since oscillation onset and offset occur through different bifurcations (the Hopf bifurcation and the cyclic fold bifurcation, respectively), this phenomenon is commonly observed as different vocal fold's configurations at voice onset and offset. For example, it is known that voice onset requires a subglottal air pressure above certain positive threshold level. However, after voice has started, the subglottal pressure may be decreased below the initial threshold, without the interruption of voice. The pressure level at which voice stops is lower than the level at which it starts $[18,19]$. This phenomenon is important to speech production, since it determines patterns of voice onset and offset during running speech (e.g., [20]). Its occurrence has been attributed to the flow-induced nature of the oscillation, and it appears to be a common phenomenon in cases of aeroelastic oscillations in nature, such as those of buildings and bridges by action of the wind, and airplane wings during flight [21].

A different large amplitude extension was recently proposed by Laje et al. [22], which kept Titze's original equations but added a nonlinear damping characteristic for the tissues. The nonlinear damping was introduced ad-hoc to limit the oscillation amplitude at large oscillations, and it would account for nonlinear effects such as pressure losses at the glottis for air viscosity, collision between the opposite folds and consequent interruption of the airflow, formation of an air jet downstream from the glottis, nonlinearities of tissue biomechanics, and other factors. A similar nonlinear damping characteristic was also used in a recent modeling work [20], based on tissue biomechanics considerations. This model is attractive in its simplicity, and has been also applied to the labia oscillation at the syrinx of songbirds [2].

The purpose of this paper is to investigate the dynamics of Laje et al.'s model [22]. Particularly, it intends to determine the type of bifurcation that produces the oscillation, and whether the model allows for the occurrence of the oscillation hysteresis phenomenon, discussed above. The following sections will first review the derivation of the model's equation. Next, its dynamical structure will be analyzed and illustrated through phase plane plots and bifurcation diagrams. The results will be summarized and discussed in a final conclusion section.

\section{Vocal fold model}

The model is illustrated in Fig. 1 [14]. For clarity of the present analysis, main steps of the derivation of the model's equation are reproduced here.

Complete right-left symmetry of the folds is assumed, and motion is allowed only in the horizontal direction. A surface wave propagates through the superficial mucosal tissues of the folds, in the direction of the airflow (upward). Letting $\xi$ be displacement of the tissues from their rest position, and $y$ the vertical distance from the midpoint of the glottis in the direction of the airflow, then the surface wave has the general expression

$$
\xi(y, t)=x(t-y / c),
$$

where $t$ is time, $x(t)=\xi(0, t)$ is the displacement of the tissues at the midpoint of the 


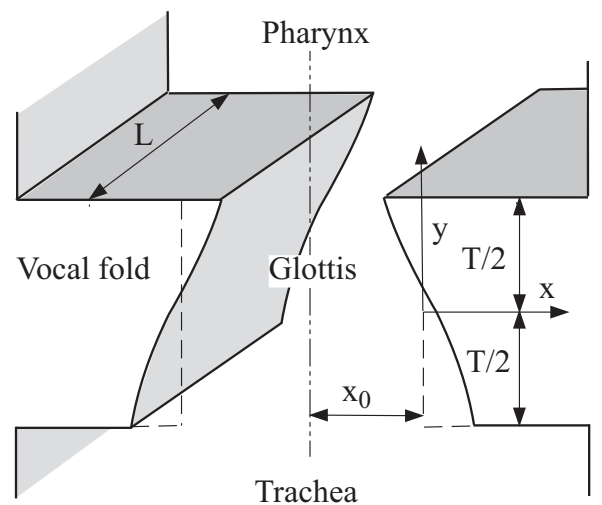

FIG. 2.1. Vocal fold model [14].

glottis and $c$ is the wave velocity. This expression is next expanded into a Taylor series around $y=0$, and approximated by the linear terms

$$
\xi(y, t) \approx x(t)-(y / c) \dot{x}(t) .
$$

The above approximation is valid assuming that the time delay $\tau=T /(2 c)$ for the surface wave to travel half the glottal height $T / 2$ is sufficiently small, compared to the period of the oscillation[14, 10].

We consider the simple case in which the vocal fold separation along the glottal height is constant, when they are at their rest position. In this case, the glottal cross sectional area $a$ at the height $y$ is $a=2 L\left(x_{0}+\xi\right)$, where $x_{0}$ is the half-width at the rest position, and $L$ is the glottal length. The glottal areas $a_{1}$ and $a_{2}$ at the lower $(y=-T / 2)$ and upper $(y=T / 2)$ edges of the vocal folds, respectively, are then approximated by

$$
\begin{aligned}
& a_{1}=2 L\left(x_{0}+x+\tau \dot{x}\right), \\
& a_{2}=2 L\left(x_{0}+x-\tau \dot{x}\right) .
\end{aligned}
$$

From the lungs up to the exit of the glottis, the air flow is assumed approximately frictionless, stationary, and incompressible [24]. Under these conditions, Bernoulli's energy equation may be used to describe the flow. The equations are further simplified by neglecting pressure losses at the bronchi and trachea and hence assuming that the subglottal pressure is constant and equal to the lung pressure $P_{L}$. Also, pressure at the exit of the glottis is assumed constant and equal to the atmospheric pressure $P_{o}=0$. These are standard assumptions to investigate the main mechanism of the vocal fold oscillation, isolated from vocal tract and subglottal influences, and approximately correspond to laboratory conditions of excised larynges [18].

Considering that the area of the trachea is much larger than the glottal area, and according to Bernoulli's equation, the pressure difference between $P_{L}$ at the exit of the trachea and $P_{2}$ at the upper edge of the vocal folds is

$$
P_{L}-P_{2}=\frac{\rho u^{2}}{2 a_{2}^{2}}
$$


where $\rho$ is the air density, and $u$ is the volume velocity of the glottal airflow. At the glottal exit, and due to the abrupt area expansion, the flow detaches from the glottal wall and forms a jet stream, losing almost all its energy by turbulence [24]. Hence, $P_{2} \approx P_{o}=0$.

The glottal pressure acting on the vocal fold medial surface is calculated as the mean glottal pressure

$$
P_{g}=\frac{1}{T} \int_{-T / 2}^{T / 2} P(y) d y,
$$

where $P(y)$ is the pressure distribution along the glottal height. This distribution may be obtained by applying again Bernoulli's equation between the position at height $y$ and the glottal exit

$$
\begin{aligned}
P(y) & =\frac{\rho u^{2}}{2}\left(\frac{1}{a_{2}^{2}}-\frac{1}{a(y)^{2}}\right), \\
& =P_{L}\left(1-\frac{a_{2}^{2}}{a(y)^{2}}\right) .
\end{aligned}
$$

Note that the approximation given by (2.2) implies that

$$
\frac{d a}{d y}=2 L \frac{d \xi}{d y} \approx-\frac{2 L}{c} \dot{x}
$$

which is independent of $y$. Hence, the assumption of a small value for $\tau$ implies a linear variation of the glottal area along the glottal height, between the lower and upper area values $a_{1}$ and $a_{2}$, respectively. In this case, computation of the integral in (2.6) yields $[14,10]$

$$
\begin{aligned}
P_{g} & =P_{L}\left(1-\frac{a_{2}}{a_{1}}\right), \\
& =\frac{2 P_{L} \tau \dot{x}}{x_{0}+x+\tau \dot{x}} .
\end{aligned}
$$

Note that (2.10) was derived considering that the glottis is open, and so the denominator must be $a_{1}>0$, or equivalently, $x_{0}+x+\tau \dot{x}>0$.

The above equations allows us to offer a simplified explanation of the vocal fold oscillation mechanics (see also [1, 14]). Note first, in (2.3) and (2.4), that when the vocal folds move away from each other $(\dot{x}>0)$ the glottal channel takes a convergent shape $\left(a_{1}>a_{2}\right)$. In this case, the area reduction causes a positive glottal pressure, as indicated by (2.9), which acts in the sense of pushing the folds apart. On the other hand, when the vocal folds move towards each other $(\dot{x}<0)$ closing the glottis, the opposite situation occurs. In this case the glottal channel is divergent $\left(a_{1}<a_{2}\right)$, and the area expansion causes a negative glottal pressure, which acts in the sense of sucking the folds together. Thus, the glottal pressure always acts in the same direction of movement as that of the folds (note in 2.10 that the glottal pressure $P_{g}$ has the same sign as the vocal fold velocity $\dot{x}$ ), which implies a transfer of energy from the airflow to the vocal fold. When this energy is enough to overcome the energy lost by dissipation in the tissues, the oscillation starts.

The mechanical properties of the vocal fold tissues are lumped at the midpoint of the glottis, which yields the equation of motion

$$
M \ddot{x}+B\left(1+\eta x^{2}\right) \dot{x}+K x=P_{g},
$$


where $M, B$, and $K$ are the mass, damping, and stiffness, respectively, per unit area of the vocal fold medial surface, and $\eta$ is a phenomenological nonlinear coefficient. This equation includes a nonlinear damping term $B\left(1+\eta x^{2}\right) \dot{x}$ instead of the usual linear one $B \dot{x}$ used by Titze [14] in his small amplitude analysis. As mentioned in $\S 1$, this term accounts for nonlinear effects of the glottal aerodynamics, tissue viscoelasticity, and vocal fold collision, which act as limiting factors of the oscillation amplitude [22]. In a previous modeling work [20], a similar term of the form $B(1+\eta|x|) \dot{x})$ was also incorporated into a vocal fold model. The adoption of such term was based on empirical data from vocal fold tissues [23], which showed that the time constant of tissue relaxation curves increases with the level of strain imposed. Such an empirical result may be modeled by a damping term which increases with the deformation of tissues, as the one proposed above. The data show larger variations of the time constant at larger levels of strain, which seems to indicate that a nonlinear term with $x^{2}$ would be more appropriate than the previous one with $|x|$. However, the available numerical data is rather limited for a better comparison.

Joining (2.11) and (2.10), we obtain finally the complete equation

$$
M \ddot{x}+B\left(1+\eta x^{2}\right) \dot{x}+K x=\frac{2 P_{L} \tau \dot{x}}{x_{0}+x+\tau \dot{x}}, \quad \text { with } x_{0}+x+\tau \dot{x}>0 .
$$

More details on the above equations may be easily found in the indicated references. Note that the differential equation (2.12) holds under an inequality constraint. The conditions for this constraint to be satisfied (or not) will be also explored in the following sections.

\section{Normalization and numerical example}

The number of parameters may be reduced by introducing the new adimensional variables $u=x / x_{0}, \nu=t \sqrt{K / M}$, and the parameters $\alpha=B / \sqrt{M K}, \beta=x_{0}^{2} \eta$, and $\gamma=$ $2 \tau P_{L} /\left(x_{0} \sqrt{M K}\right), \delta=\tau \sqrt{K / M}$ which yields the differential equation

$$
u^{\prime \prime}+\alpha\left(1+\beta u^{2}\right) u^{\prime}+u=\frac{\gamma u^{\prime}}{1+u+\delta u^{\prime}}, \quad \text { with } 1+u+\delta u^{\prime}>0,
$$

and $u^{\prime}=d u / d \nu$. Letting $v=u^{\prime}$, the following equivalent bidimensional form is obtained

$$
\left\{\begin{array}{l}
u^{\prime}=v, \\
v^{\prime}=-\alpha\left(1+\beta u^{2}\right) v-u+\frac{\gamma v}{1+u+\delta v}, \quad \text { with } 1+u+\delta v>0 .
\end{array}\right.
$$

Typical adult values for the parameters are $c=100 \mathrm{~cm} / \mathrm{s}, T=3 \mathrm{~mm}, x_{0}=1 \mathrm{~mm}$, $M=0.476 \mathrm{~g} / \mathrm{cm}^{2}, B=100$ dyne s $/ \mathrm{cm}^{3}, K=200000$ dyne $/ \mathrm{cm}^{3}$ [14]. For a numerical example, we consider $P_{L}=8000$ dyne $/ \mathrm{cm}^{2}$, which is the lung pressure of an adult's normal voice [1], and $\eta=1000 \mathrm{~cm}^{-2}$. The adimensional parameters are then $\alpha=0.32$, $\beta=100, \gamma=0.78, \delta=0.97$.

Fig. 3.1 shows a phase portrait for the above parameters, with six trajectories. The dashed line corresponds to the singular condition $1+u+\delta v=0$, and marks the limit of validity of (3.2). At this condition, the opposite vocal folds become in contact and close the glottis, interrupting the airflow. Trajectory 1 is the normal case of phonation. The rest position at $(u, v)=(0,0)$ is unstable, which causes an oscillation of increasing amplitude, until reaching a limit cycle. Trajectories 2, 3, and 4 are outside the limit cycle, but still within its basin of attraction. Trajectory 5 marks the limit of the basin of attraction of the limit cycle. Note that this trajectory tends to 


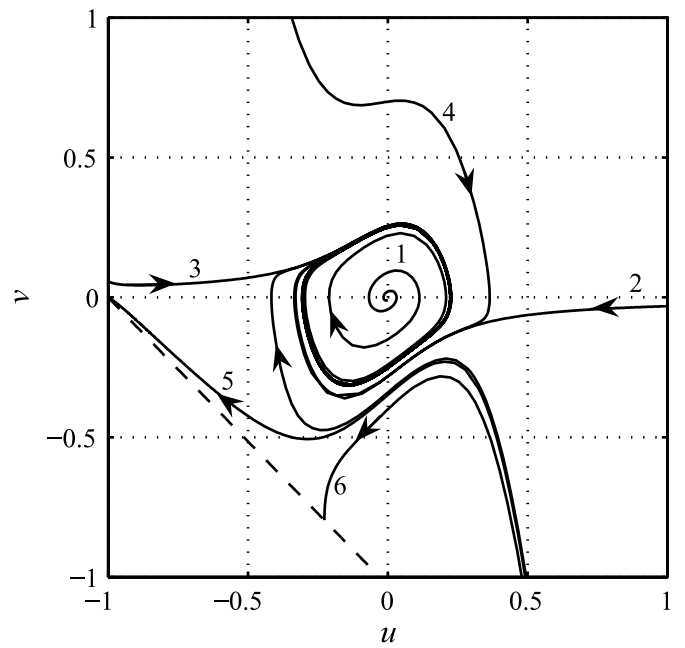

FIG. 3.1. A phase portrait for Eq. 3.2, and $\alpha=0.32, \beta=100, \gamma=0.78, \delta=0.97$.

the singular point $(-1,0)$, at which both the numerator and denominators at the left side of (3.2) become zero. Trajectory 6 is outside the basin of attraction of the limit cycle. This trajectory, as well as the whole region below trajectory 5, are outside the region of validity of the model as a representation of the vocal fold oscillator, since they do not correspond to licit vocal fold motion. The phase portrait duplicates results obtained by Laje et al.'s [22]. In the next section, the formation of the limit cycle is analyzed in more detail.

\section{Stability of the rest position and Hopf bifurcation}

The dynamical structure of the above model will be explored considering $\gamma$ as control parameter. This parameter is directly related to the lung pressure, which is the main parameter to control voicing offset and onset, as well as voice intensity [1].

Equation (3.2) has one equilibrium position at $(u, v)=(0,0)$. The eigenvalues $\lambda$ of the Jacobian matrix at this position are given by the characteristic equation

$$
\lambda^{2}+(\alpha-\gamma) \lambda+1=0 .
$$

This equation has a pair of conjugate complex roots, which crosses the imaginary axis from left to right as $\gamma$ increases and crosses the bifurcation value $\gamma=\alpha$. At this value, a Hopf bifurcation occurs, in which the equilibrium position changes its stability: it is a stable focus for $\gamma<=\alpha$, and an unstable one at $\gamma>\alpha$. At the same time, a limit cycle is generated [25].

Let us recall that there are two types of Hopf bifurcation: the supercritical case, in which a stable limit cycle is expelled from the equilibrium position, and the subcritical case, in which an unstable limit cycle is absorbed. To determine the type of Hopf bifurcation, we may follow Perko's version of Hopf's bifurcation Theorem for planar systems [25]: Let $s$ be the signed distance along a line through the origin, and $P(s)$ be the Poincaré map for the focus. Further, let $\sigma \equiv d^{\prime \prime \prime}(0)$ be the Lyapunov number for the focus, where $d(s)=P(s)-s$ is the displacement function. Then, if $\sigma \neq 0$, the origin is a weak focus and a Hopf bifurcation occurs at the bifurcation value of the 
control parameter, and the sign of $\sigma$ indicates its type: it is supercritical for $\sigma<0$, and subcritical for $\sigma>0$. For a general planar analytic system

$$
\begin{aligned}
& \dot{x}=a x+b y+p(x, y), \\
& \dot{y}=c x+d y+q(x, y),
\end{aligned}
$$

where $\Delta=a d-b c>0, a+d=0$, and the analytic functions $p(x, y)=\sum_{i+j \geq 2} a_{i j} x^{i} y^{j}$, $q(x, y)=\sum_{i+j \geq 2} b_{i j} x^{i} y^{j}$, the origin is a weak focus and the Lyapunov number is given by

$$
\begin{gathered}
\sigma=\frac{-3 \pi}{2 b \Delta^{3 / 2}}\left\{\left[a c\left(a_{11}^{2}+a_{11} b_{02}+a_{02} b_{11}\right)+a b\left(b_{11}^{2}+a_{20} b_{11}+a_{11} b_{02}\right)\right.\right. \\
+c^{2}\left(a_{11} a_{02}+2 a_{02} b_{02}\right)-2 a c\left(b_{02}^{2}-a_{20} a_{02}\right)-2 a b\left(a_{20}^{2}+b_{20} b_{02}\right) \\
\left.-b^{2}\left(2 a_{20} b_{20}+b_{11} b_{20}\right)+\left(b c-2 a^{2}\right)\left(b_{11} b_{02}-a_{11} a_{20}\right)\right] \\
\left.-\left(a^{2}+b c\right)\left[3\left(c b_{03}-b a_{30}\right)+2 a\left(a_{21}+b_{12}\right)+\left(c a_{12}-b b_{21}\right)\right]\right\} .
\end{gathered}
$$

In our case, for $\gamma=\alpha$, we have

$$
\left\{\begin{array}{l}
u^{\prime}=v \\
v^{\prime}=-u-\alpha v u-\alpha \delta v^{2}-\alpha(\beta-1) u^{2} v+2 \alpha \delta u v^{2}+\alpha \delta^{2} v^{3}+\ldots
\end{array}\right.
$$

which produces

$$
\sigma=\frac{3 \pi}{2} \alpha\left(1-\beta+\alpha \delta+3 \delta^{2}\right)
$$

Hence, for $1-\beta+\alpha \delta+3 \delta^{2}<0(\sigma<0)$ the Hopf bifurcation is supercritical, and for $1-\beta+\alpha \delta+3 \delta^{2}>0(\sigma>0)$ it is subcritical.

\section{Continuation analysis}

The characteristics of the limit cycle and bifurcations in the system may be explored using the software package MATCONT [26]. This package is a collection of numerical algorithms implemented as a MATLAB Toolbox, for detection and continuation of equilibria, limit cycles and bifurcations in differential equations.

Fig. 5.1 shows the limit cycle generated at the Hopf bifurcation, when varying $\gamma$, with the other parameters at their previous typical values of $\S 4$. As this $\gamma$ crosses its bifurcation value and increases, a stable limit cycle is expelled from the origin, and grows in amplitude. In this case, (4.5) produces $\sigma=-144.5<0$, indicating a supercritical bifurcation.

It is interesting to see how the dynamics of the system change for different values of $\beta$. The other parameters, $\alpha$ and $\delta$, depend on physiological properties of the vocal fold tissues such as mass, stiffness, and damping ratio, which are relatively well known. Parameter $\beta$, on the other hand, is related to the phenomenological parameter $\eta$, which accounts for several nonlinear effects at large amplitude oscillations, and hence its value is more uncertain. Further, it is also proportional to $x_{0}$ (vocal fold separation), which is a parameter actively used by speakers to control voicing onset and offset in consonant production during speech (see, e.g., [20]). Parameters $\alpha$ and $\delta$, should be more constant during speech, except for variations to control voice pitch (oscillation frequency).

Fig. 5.2 shows limit cycle curves for several values of $\beta$. Point $H$ denotes the Hopf bifurcation. Curves growing to the right from $\mathrm{H}$ represent stable limit cycles, and 


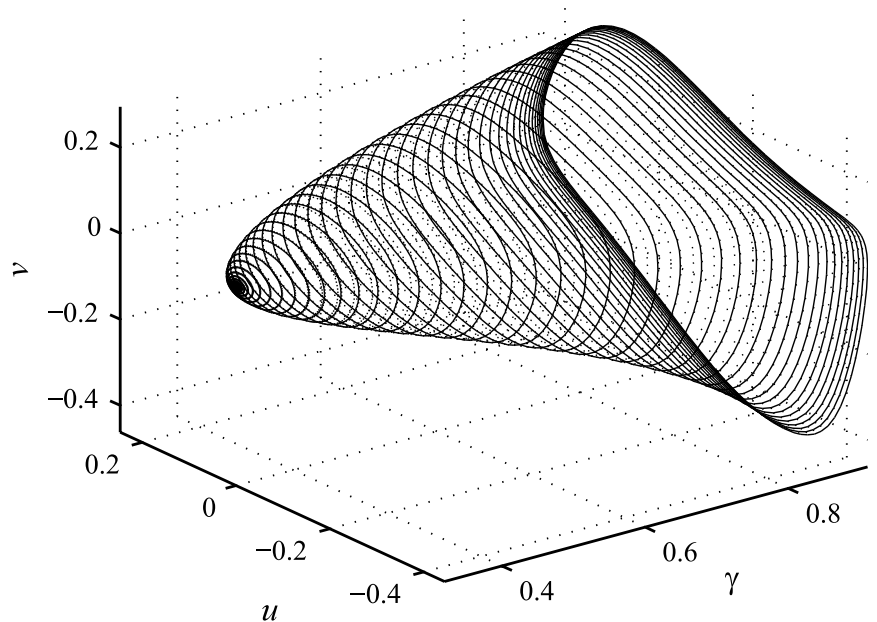

FIG. 5.1. Limits cyle vs. $\gamma$, and $\alpha=0.32, \beta=100, \delta=0.97$.

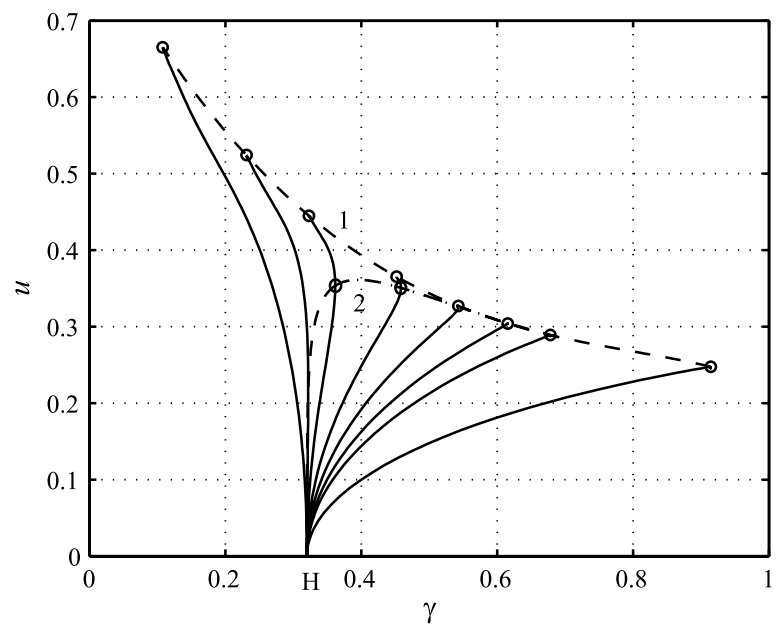

FIG. 5.2. Amplitude of limit cycles vs. $\gamma$, for $\alpha=0.32, \delta=0.97$, and various values of $\beta$. The vertical axis is the maximum amplitude of $u$ for the limit cycle. From left to right, the curves correspond to values of $\beta=0.1,5,10,20,30,40,50,100$. Dashed line 1 is the maximum amplitude that the limit cycle might reach. Dashed line 2 is the location of a cyclic fold bifurcation.

the bifurcation is supercritical. Those growing from $\mathrm{H}$ to the left represent unstable cycles, and the bifurcation is subcritical. The amplitude of the limit cycles may grow until reaching line 1. Beyond this point, trajectories starting near the origin spiral out and get attracted to the singular point at $(-1,0)$, which prevents the formation of the limit cycle. Fig. 5.3 shows an example of such case.

Some curves growing to the right from $\mathrm{H}$ change direction and turn to the left at larger amplitudes. The point of direction inversion represents a fold bifurcation between limit cycles. The portion of the curve growing to the right represents a 


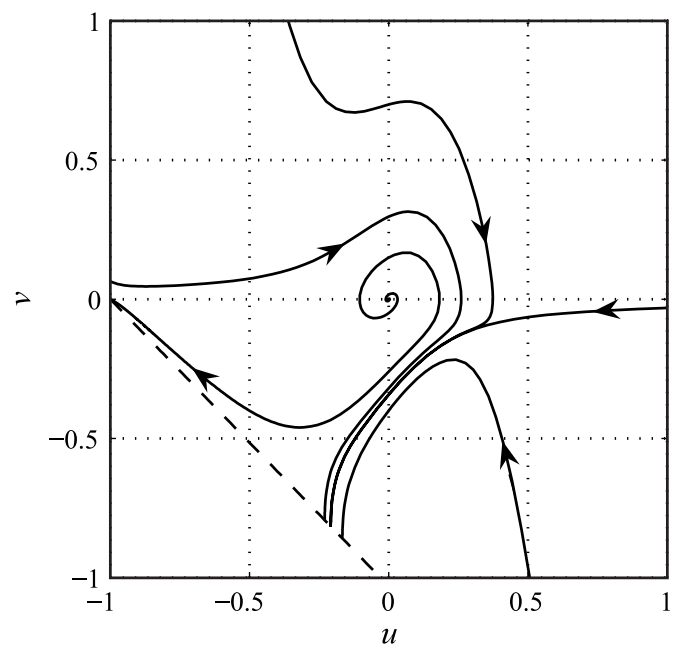

FIG. 5.3. Phase portrait for $\gamma=1, \beta=100, \alpha=0.32, \delta=0.97$.

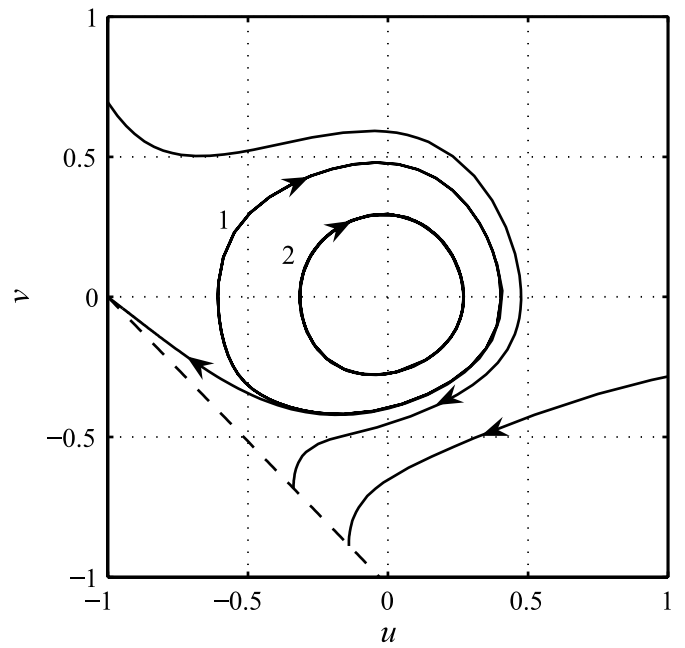

FIG. 5.4. Phase portrait for $\gamma=0.35, \beta=10, \alpha=0.32, \delta=0.97$. Limit cycle 1 is unstable, and limit cycle 2 is stable.

stable limit cycle, and the upper portion growing to the left represents an unstable limit cycle. Thus, for some values of $\gamma$, both limit cycles may co-exist, as shown in Fig. 5.4. As $\gamma$ increases, both limit cycles coalesce and cancel each other.

Fig. 5.2 also shows an important fact: in the case of a subcritical bifurcation (curves growing to the left from $\mathrm{H}$ ), there is no stable limit cycle for any value of $\gamma$. See an example in Fig. 5.5. The range of parameters which allow the subcritical bifurcation is then outside the region of validity of the model as a representation of the vocal fold oscillator. Therefore, this model can not reproduce the oscillation hysteresis phenomenon discussed in $\S 1$, which requires a subcritical bifurcation. Let us note 


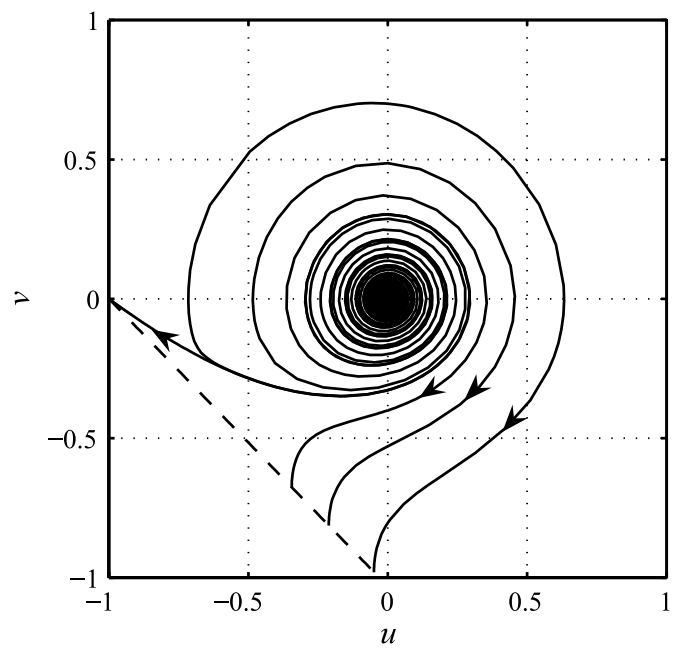

FIG. 5.5. Phase portrait for $\gamma=0.4, \beta=1, \alpha=0.32, \delta=0.97$.

that this conclusion holds for the adopted values of parameters $\alpha=0.32$ and $\delta=0.97$, which were kept fixed along the analysis. It is not clear whether other values for those parameters would lead to a different result, which certainly poses an interesting question for further research. However, let us also recall from $\S 3$ that the adopted values are typical for a normal adult larynx [14]. Thus, the fact that the model can not reproduce hysteresis patterns in such configuration limits its application to voice production studies.

\section{Modification of the model}

A possible way to allow for both a subcritical bifurcation and a stable limit cycle is to augment the model by adding higher powers of $u$ to the damping factor in (3.1). The shape of the curves that represent the limit cycle amplitudes (as those shown in Fig. 5.2) may be easily altered by using suitable even-powered polynomials. This approach has been used to model subcritical Hopf bifurcations and oscillation hysteresis in other mechanical systems, such as axial flow compressors [27]. Here, the simple modification of (3.1) is considered

$$
u^{\prime \prime}+\alpha\left(1+\beta_{1} u^{2}+\beta_{2} u^{4}\right) u^{\prime}+u=\frac{\gamma u^{\prime}}{1+u+\delta u^{\prime}}, \quad \text { with } 1+u+\delta u^{\prime}>0 .
$$

The Lyapunov number is given by an expression similar to (4.5), substituting $\beta_{1}$ in place of $\beta$. The bifurcation is then subcritical $(\sigma>0)$ for small values of $\beta_{1}$.

Fig. 6.1 shows plots of limit cycle continuations, for $\beta_{1}=0$ and various values of $\beta_{2}$. As in Fig. 5.2, line 1 indicates the maximum amplitude of the limit cycle allowed by the system, and line 2 is a continuation of the cyclic fold between limit cycles of opposite stability.

Fig. 6.2 shows curve for $\beta_{2}=200$ in more detail. $\mathrm{H}$ denotes the Hopf bifurcation and $\mathrm{CF}$ the cyclic fold bifurcation. The dotted line represents an unstable limit cycle, produced at the Hopf bifurcation, which is of the subcritical type. This limit cycle coalesces and cancels out with a stable limit cycle, indicated by the full line, at the cyclic fold. 


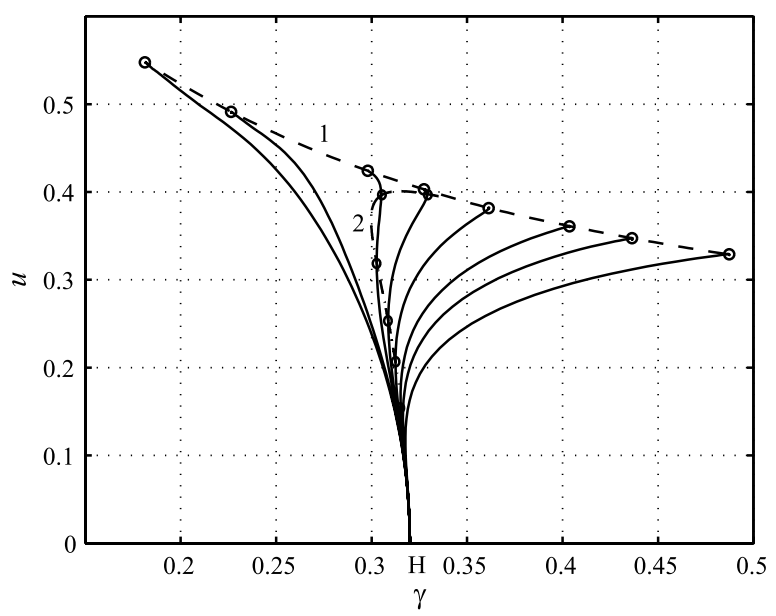

FIG. 6.1. Amplitude of limit cycles vs. $\gamma$, for $\beta_{1}=0$ and various values of $\beta_{2}$. The vertical axis is the maximum amplitude of $u$ for the limit cycle. From left to right, the curves correspond to values of $\beta_{2}=10,20,50,70,100,150,200,300$. Dashed line 1 is the maximum amplitude that the limit cycle might reach. Dashed line 2 is the location of a cyclic fold bifurcation.

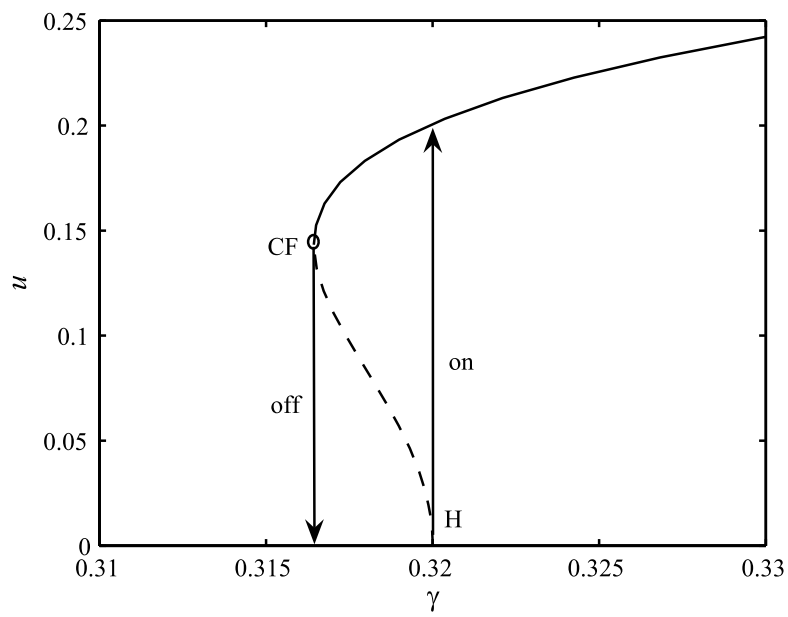

FIG. 6.2. Amplitude of limit cycles vs. $\gamma$, for $\beta_{1}=0$ and $\beta_{2}=200$. The vertical axis is the maximum amplitude of $u$ for the limit cycle. The full line represents a stable limit cycle, and the dashed line represents an unstable limit cycle.

Fig. 6.2 also shows the occurrence of the oscillation hysteresis phenomenon. Let us assume that the system is at its rest position in $(0,0)$ and that control parameter $\gamma$ increases from zero. This is the common case at the start of phonation, when the vocal folds are set at rest at their prephonatory position, and the lung pressure builds up from zero. When $\gamma$ reaches its Hopf bifurcation value $(\gamma=\alpha)$, the rest position becomes unstable, and an oscillatory motion starts. This is represented by the "on" arrow in Fig. 6.2. The oscillatory motion grows in amplitude until stabilizing in a limit cycle (at the full line curve). Further increase of $\gamma$ causes the limit cycle to increase 
in amplitude, following the full curve to the right. Next, assume that $\gamma$ is decreased, with the intention of stopping the oscillation. The limit cycle decreases in amplitude following its curve to the left. However, the limit cycle will vanish only when reaching the cyclic fold bifurcation at CF. At this point, the oscillation will damp out and the system will return to its rest position, as represented by the "off" arrow.

In this way, the oscillation onset and offset occur at different values of the control parameter $\gamma$, creating a hysteresis loop. As discussed in $\S 1$, this phenomenon has been observed in several experimental studies of voicing onset-offset. For example, recent direct measurements of phonation threshold pressures in subjects has detected lower values at offset vs. onset for adult speakers performing a number of speech tasks [19]. It must be noted that those measurements show differences between onset and offset pressures much larger than the one shown in Fig. 6.2. In some cases, the offset value is about half the onset value. However, let us keep in mind that the model under study is a gross simplification of the actual vocal fold biodynamics, and hence precise quantitative predictions must not be expected. In comparison, the previous large amplitude model $[15,10]$, which included a more detailed description of the glottal aerodynamics, has been able to produce offset/onset pressure ratios from 0.5 to 1 , which is in the range of the experimental results.

\section{Conclusion}

This paper has investigated the dynamics of a simple bidimensional model for the vocal fold oscillation at phonation. The model is capable of producing an oscillation with physiologically realistic values for the parameters, and hence may be used as a valid representation for the vocal folds in studies of voice production. Simple extensions of the model using even-powered polynomials in the damping factor allow for the reproduction of more subtle details, such as the oscillation hysteresis phenomenon commonly observed in voice onset-offset patterns.

The model must be used with caution, perhaps limited to qualitative studies surrounding the oscillation onset and offset, rather than full simulations of the vocal fold oscillation. The actual oscillation includes more complex phenomena such as collision between the opposite folds, air viscous effects when the folds are close together, formation of air jets and vortices at the glottal exit, and other effects which have not been considered here. More sophisticated models must be used, when pretending quantitative predictions of voice production.

\section{REFERENCES}

[1] I. R. Titze, Principles of Voice Production, Prentice-Hall, Englewood Cliffs, 1994.

[2] R. Laje, T. J. Gardner and G. B. Mindlin, Neuromuscular control of vocalizations in birdsong: A model, Phys. Rev. E, 65, 051921, 2002.

[3] O. E. Jensen, Instabilities of flow in a collapsible tube, J. Fluid Mech., 220, 623-659, 1990.

[4] S. Adachi and M. A. Sato, Trumpet sound simulation using a two-dimensional lip vibration model, J. Acoust. Soc. Am., 99, 1200-1209, 1996.

[5] J. W. van den Berg, J. T. Zantema and P. Doornenbal, On the air resistance and the Bernoulli effect of the human larynx, J. Acoust. Soc. Am., 29, 626-631, 1957.

[6] J. L. Flanagan and L. L. Landgraf, Self-oscillating source for vocal-tract synthesis, IEEE Trans. Audio Eletroacoust., AU-16, 57-64, 1968.

[7] K. Ishizaka and J. L. Flanagan, Synthesis of voiced sounds from a two-mass model of the vocal cords, Bell Syst. Tech. J., 51, 1233-1268, 1972.

[8] F. Alipour, D. Berry and I. R. Titze, A finite-element model of vocal fold vibration, J. Acoust. Soc. Am., 108, 3003-3012, 2000.

[9] C. E. Vilain, X. Pelorson, C. Fraysse, M. Deverge, A. Hirschberg and J. Willems, Experimental 
validation of a quasi-steady theory for the flow through the glottis, J. Sound Vibration, $276,475-490,2004$

[10] J. C. Lucero, A theoretical study of the hysteresis phenomenon at vocal fold oscillation onsetoffset, J. Acoust. Soc. Am., 105, 423-431, 1999.

[11] H. Herzel and C. Knudsen, Bifurcation in a vocal fold model, Nonlinear Dyn., 7, 53-64, 1995.

[12] M. A. Trevisan, M. C. Eguia and G. B Mindlin, Nonlinear aspects of analysis and synthesis of speech time series data, Phys. Rev. E, 63, 026216, 2001.

[13] J. J. Jiang, Y. Zhang and J. Stern, Modeling of chaotic vibrations in symmetric vocal folds, J. Acoust. Soc. Am., 110, 2120-2128, 2001

[14] I. R. Titze, The physics of small-amplitude oscillation of the vocal folds, J. Acoust. Soc. Am., 83, 1536-1552, 1988.

[15] J. C. Lucero, A subcritical Hopf bifurcation at phonation onset, J. Sound Vibration, 218, 344349, 1998

[16] E. V. Appleton and B. van der Pol, On a type of oscillation-hysteresis in a simple triode generator, Philosophical Magazine, 43, 177-193, 1922.

[17] N. Minorsky, Nonlinear Oscillations, Robert E. Krieger, Malabar, 1962.

[18] D. A. Berry, H. Herzel, I. R. Titze and B. H. Story, Bifurcations in excised larynx experiments, J. Voice, 10, 129-138, 1996.

[19] R. L. Plant, G. L. Freed and R. E. Plant, Direct measurements of onset and offset phonation threshold pressure in normal subjects, J. Acoust. Soc. Am., 1116, 3640-3646, 2004.

[20] J. C. Lucero and L. L. Koenig, Simulations of temporal patterns of oral airflow in men and women using a two-mass model of the vocal folds under dynamic control, J. Acoust. Soc. Am. 117, 1362-1372, 2005.

[21] I. M. T. Thompson and H. B. Stewart, Nonlinear Dynamics and Chaos, John Wiley and Sons, New York, 1987.

[22] R. Laje, T. J. Gardner and G. B. Mindlin, Continuous model for vocal fold oscillations to study the effect of feedback, Phys. Rev. E, 64, 056201, 2001.

[23] F. Alipour-Haghighi and I. R. Titze, Viscoelastic modeling of canine vocalis muscle in relaxation, J. Acoust. Soc. Am., 78, 1939-1943, 1985.

[24] X. Pelorson, A. Hirschberg, R. R. van Hassel and A. P. J. Wijnands, Theoretical and experimental study of quasisteady-flow separation within the glottis during phonation. Application to a modified two-mass model, J. Acoust. Soc. Am., 96, 3416-3431, 1994.

[25] L. Perko, Differential Equations and Dynamical Systema, Springer-Verlag, New York, 1991.

[26] A. Dhooge, W. Govaerts and Y. A. Kuznetsov, MATCONT : A Matlab package for numerical bifurcation analysis of ODEs, ACM Transactions on Mathematical Software, 29, 141-164, 2003.

[27] N. Ananthkrishnan, K. Sudhakar, S. Sudershan and A. Agarwal, Application of secondary bifurcations to large amplitude limit cycles in mechanical systems, J. Sound Vibration, $215,183-188,1998$ 